\title{
Crystal Structure of Pigment Yellow 138 Derivative
}

\author{
Isao FuJII, ${ }^{* \dagger}$ Tomohiro Kodama, $* *$ Naoto Yanagihara, $* *$ and Noriaki Hirayama*** \\ *Department of Biological Science and Technology, Tokai University, Numazu, Shizuoka 410-0321, Japan \\ **Fujinomiya Research Laboratories, Fuji Photo Film Co. Ltd., Fujinomiya, Shizuoka 418-8666, Japan \\ ***School of Medical Science, Tokai University, Isehara, Kanagawa 259-1121, Japan
}

\begin{abstract}
An X-ray analysis of the title pigment was undertaken to understand the structure-stability relationships. The molecule takes a non-planar structure, because of the 2-(inden-2-yl)quinoline and isoindoline fragments being twisted at the C8-N21 bond. The dihedral angles between three planar moieties are 4.2(3) and 64.4(3) ${ }^{\circ}$ In the crystal structure, the flat 2-(inden-2-yl)quinoline fragments are stacked opposed to each other. The isoindoline rings sandwich the solvent molecules. The solvent molecule sits on a crystallographic center of symmetry, and is disordered in the solvent channels along the c-axis.
\end{abstract}

(Received December 16, 2003; Accepted February 12, 2004; Published on Web April 23, 2004)

The title compound, 2-(1,2-dihydro-2-(1,3-dioxo- $1 H$-inden$2(3 H)$-ylidene)quinolin-8-yl)isoindoline-1,3-dione, $(\mathbf{I})$, is a type of quinophtalone yellow. A series of the pigments are manufactured on a large scale, and are of significant commercial importance worldwide, as well as C.I. Pigment Yellow 138 (PY-138); CAS No. 30125-47-4, (II). To understand the structure-stability relationships, we conducted an X-ray analysis of the title compound.

The dechlorinated compound (I), which consists of the skeleton of PY-138, was prepared by a modifying method

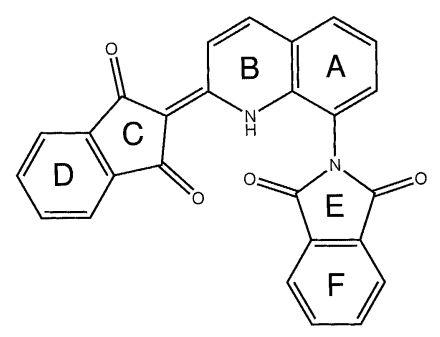

(I)

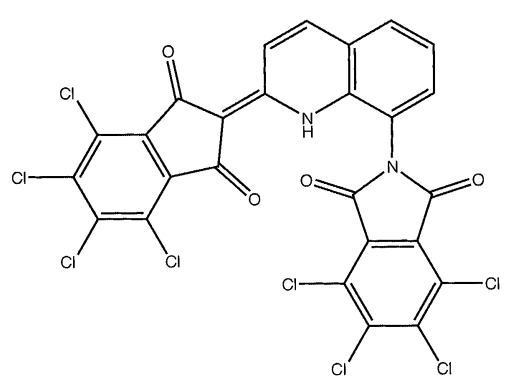

(II)

Fig. 1 Chemical structure.

† To whom correspondence should be addressed. E-mail address: fujii@wing.ncc.u-tokai.ac.jp reported by Malanker et al. ${ }^{1}$ from 8 -amino-2-methylquinoline and phthalic anhydride, purchased from Tokyo Chemical Industry (Tokyo). Crystals were obtained from a toluene solution with slow evaporation at room temperature. A platelike yellow crystal of $0.4 \times 0.4 \times 0.2 \mathrm{~mm}$ in size was used for diffraction experiments.

The crystal and experimental data are given in Table 1 . The structure was solved by direct methods using the program SIR $88,{ }^{2}$ and refined by full-matrix least-squares. Non-hydrogen atoms, except for the solvent molecule, were refined anisotropically. The solvent molecule was disordered, and the coordinates of all atoms were rigidly refined and finally fixed. All hydrogen atoms of the pigment molecule were determined from difference Fourier maps and refined isotropically. The atomic parameters for the non-hydrogen atoms are listed in

Table 1 Crystal and experimental data

Formula: $\mathrm{C}_{26} \mathrm{H}_{14} \mathrm{~N}_{2} \mathrm{O}_{4} \cdot 0.5\left(\mathrm{C}_{7} \mathrm{H}_{8}\right)$

Formula weight: 464.48

Crystal system: triclinic

Space group: $P \overline{1} \quad Z=2$

$a=12.436(4) \AA$

$b=12.564(4) \AA$

$c=7.781(5) \AA$

$\alpha=107.48(4)^{\circ}$

$\beta=92.14(4)^{\circ}$

$\gamma=79.931(3)^{\circ}$

$V=1141.7(9) \AA^{3}$

$D_{\mathrm{x}}=1.35 \mathrm{~g} / \mathrm{cm}^{3}$

No. of reflections used $=4702$

$2 \theta_{\max }=149.9^{\circ}$ with $\mathrm{Cu} \mathrm{K}_{\alpha}$

$R=0.077$

$(\Delta / \sigma)_{\max }=0.0001$

$(\Delta \rho)_{\max }=0.39 \mathrm{e}^{-3}$

$(\Delta \rho)_{\min }=-0.32 \mathrm{e}^{-3}$

Measurement: Enraf-Nonius CAD4

Program system: teXsan

Structure determination: direct method (SIR88)

Refinement: full-matrix least-squares 
Table 2 Atomic coordinates and equivalent isotropic thermal parameters $\left(B_{\text {eq }}\right)$

\begin{tabular}{lcccc}
\hline Atom & $x$ & $y$ & $z$ & $B_{\mathrm{eq}} / \AA^{2}$ \\
\hline $\mathrm{C} 11$ & $-0.0634(2)$ & $-0.1454(1)$ & $0.5919(3)$ & $5.08(4)$ \\
$\mathrm{O} 13$ & $-0.1358(2)$ & $0.2545(2)$ & $0.7884(3)$ & $6.10(5)$ \\
$\mathrm{O} 20$ & $-0.3348(2)$ & $-0.2440(2)$ & $0.6209(3)$ & $6.07(5)$ \\
$\mathrm{O} 22$ & $-0.2139(2)$ & $-0.3803(2)$ & $0.0374(3)$ & $5.89(5)$ \\
$\mathrm{N} 1$ & $-0.2523(2)$ & $-0.0586(2)$ & $0.4734(3)$ & $4.30(5)$ \\
$\mathrm{N} 21$ & $-0.2847(2)$ & $-0.2842(2)$ & $0.3235(3)$ & $4.25(5)$ \\
$\mathrm{C} 2$ & $-0.2295(2)$ & $0.0453(2)$ & $0.5571(4)$ & $4.25(5)$ \\
$\mathrm{C} 3$ & $-0.3067(2)$ & $0.1398(2)$ & $0.5341(4)$ & $4.87(6)$ \\
$\mathrm{C} 4$ & $-0.3936(2)$ & $0.1212(2)$ & $0.4296(4)$ & $4.98(6)$ \\
$\mathrm{C} 5$ & $-0.5003(2)$ & $-0.0139(3)$ & $0.2207(4)$ & $5.35(7)$ \\
$\mathrm{C} 6$ & $-0.5116(3)$ & $-0.1229(3)$ & $0.1336(5)$ & $5.88(8)$ \\
$\mathrm{C} 7$ & $-0.4396(2)$ & $-0.2129(3)$ & $0.1674(4)$ & $5.38(7)$ \\
$\mathrm{C} 8$ & $-0.3558(2)$ & $-0.1925(2)$ & $0.2842(4)$ & $4.44(6)$ \\
$\mathrm{C} 9$ & $-0.3391(2)$ & $-0.0817(2)$ & $0.3670(3)$ & $4.21(5)$ \\
$\mathrm{C} 10$ & $-0.4137(2)$ & $0.0101(2)$ & $0.3377(4)$ & $4.52(6)$ \\
$\mathrm{C} 11$ & $-0.0562(2)$ & $-0.0451(2)$ & $0.6645(4)$ & $4.38(6)$ \\
$\mathrm{C} 12$ & $-0.1338(2)$ & $0.0536(2)$ & $0.6552(4)$ & $4.37(6)$ \\
$\mathrm{C} 13$ & $-0.0936(2)$ & $0.1551(2)$ & $0.7591(4)$ & $4.67(6)$ \\
$\mathrm{C} 14$ & $0.0847(3)$ & $0.1781(3)$ & $0.9415(4)$ & $5.54(7)$ \\
$\mathrm{C} 15$ & $0.1779(3)$ & $0.1188(3)$ & $0.9981(5)$ & $6.09(8)$ \\
$\mathrm{C} 16$ & $0.1978(3)$ & $0.0014(3)$ & $0.9438(5)$ & $6.06(8)$ \\
$\mathrm{C} 17$ & $0.1282(2)$ & $-0.0612(3)$ & $0.8347(4)$ & $5.30(7)$ \\
$\mathrm{C} 18$ & $0.0346(2)$ & $-0.0022(2)$ & $0.7783(4)$ & $4.59(6)$ \\
$\mathrm{C} 19$ & $0.0141(2)$ & $0.1157(2)$ & $0.8336(4)$ & $4.79(6)$ \\
$\mathrm{C} 20$ & $-0.2838(2)$ & $-0.3054(2)$ & $0.4893(4)$ & $4.55(6)$ \\
$\mathrm{C} 22$ & $-0.2221(2)$ & $-0.3752(2)$ & $0.1926(4)$ & $4.46(6)$ \\
$\mathrm{C} 23$ & $-0.1063(3)$ & $-0.5609(2)$ & $0.2228(5)$ & $5.73(7)$ \\
$\mathrm{C} 24$ & $-0.0756(3)$ & $-0.6212(3)$ & $0.3425(5)$ & $6.29(8)$ \\
$\mathrm{C} 25$ & $-0.1113(3)$ & $-0.5812(3)$ & $0.5187(6)$ & $6.42(9)$ \\
$\mathrm{C} 26$ & $-0.1795(3)$ & $-0.4760(3)$ & $0.5849(5)$ & $5.63(8)$ \\
$\mathrm{C} 27$ & $-0.2099(2)$ & $-0.4160(2)$ & $0.4642(4)$ & $4.54(6)$ \\
$\mathrm{C} 28$ & $-0.1738(2)$ & $-0.4565(2)$ & $0.2882(4)$ & $4.59(6)$ \\
$\mathrm{C} 29$ & 0.4509 & -0.4523 & -0.1418 & $18.9(4)$ \\
$\mathrm{C} 30$ & 0.3964 & -0.4395 & 0.0263 & $16.2(3)$ \\
$\mathrm{C} 31$ & 0.4464 & -0.4871 & 0.1671 & $19.7(4)$ \\
$\mathrm{C} 32$ & 0.3776 & -0.3895 & -0.2645 & 30.0 \\
\hline
\end{tabular}

$B_{\mathrm{eq}}=(4 / 3) \Sigma_{i} \Sigma_{j} \beta_{i j}\left(\boldsymbol{a}_{i} \cdot \boldsymbol{a}_{j}\right)$.

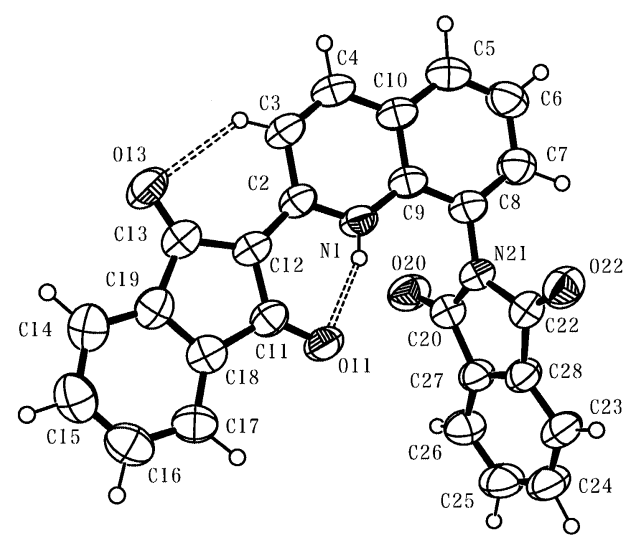

Fig. 2 ORTEP-3 drawing representing heavy atoms as 50\% probability ellipsoids and $\mathrm{H}$ atoms as circles of arbitrary size. The dashed bonds show intramolecular hydrogen bonds.

Table 2. All calculations were carried out with the program package teXsan. ${ }^{3}$

An ORTEP- $3^{4}$ drawing of the compound (I) together with the atomic numbering is shown in Fig. 2. The ring-labeling system is shown in Fig. 1. Selected bond distances, bond angles and torsion angles are given in Table 3. The molecule takes a nonplanar structure, because of two planar fragments (2-(inden-2yl)quinoline A - D and isoindoline EF) being twisted at the
Table 3 Selected bond distances $(\AA)$, bond angles $\left({ }^{\circ}\right)$ and torsion angles $\left({ }^{\circ}\right)$

\begin{tabular}{|c|c|c|c|}
\hline Bond & distances $(\AA)$ & Bond & distances (Å) \\
\hline O11-C11 & $1.234(3)$ & O13-C13 & $1.223(3)$ \\
\hline $\mathrm{O} 20-\mathrm{C} 20$ & $1.204(3)$ & $\mathrm{O} 22-\mathrm{C} 22$ & $1.198(3)$ \\
\hline $\mathrm{N} 1-\mathrm{C} 2$ & $1.347(3)$ & N1-C9 & $1.355(4)$ \\
\hline N21-C8 & $1.428(3)$ & $\mathrm{N} 21-\mathrm{C} 20$ & $1.392(4)$ \\
\hline $\mathrm{N} 21-\mathrm{C} 22$ & $1.414(3)$ & $\mathrm{C} 2-\mathrm{C} 3$ & $1.442(4)$ \\
\hline $\mathrm{C} 2-\mathrm{C} 12$ & $1.392(4)$ & C3-C4 & $1.339(4)$ \\
\hline $\mathrm{C} 4-\mathrm{C} 10$ & $1.426(4)$ & $\mathrm{C} 5-\mathrm{C} 6$ & $1.363(5)$ \\
\hline C5-C10 & $1.398(4)$ & $\mathrm{C} 6-\mathrm{C} 7$ & $1.400(4)$ \\
\hline C7-C8 & $1.364(4)$ & $\mathrm{C} 8-\mathrm{C} 9$ & $1.396(4)$ \\
\hline C9-C10 & $1.418(3)$ & C11-C12 & $1.450(4)$ \\
\hline C12- C13 & $1.450(4)$ & & \\
\hline Bond angles & $\left({ }^{\circ}\right)$ & Bond angles & $\left({ }^{\circ}\right)$ \\
\hline C2-N1-C9 & $125.9(2)$ & C8-N21-C20 & $123.4(2)$ \\
\hline C8-N21-C22 & $124.3(2)$ & C20-N21-C22 & $111.3(2)$ \\
\hline N1-C2-C3 & $116.4(3)$ & N1-C2-C12 & $118.4(2)$ \\
\hline C3-C2-C12 & $125.2(3)$ & N21-C8-C7 & $120.3(2)$ \\
\hline N21-C8-C9 & $119.4(2)$ & C7-C8-C9 & $120.3(3)$ \\
\hline $\mathrm{C} 2-\mathrm{C} 12-\mathrm{C} 11$ & $122.5(2)$ & $\mathrm{C} 2-\mathrm{C} 12-\mathrm{C} 13$ & $128.3(2)$ \\
\hline $\mathrm{C} 11-\mathrm{C} 12-\mathrm{C} 13$ & $109.2(2)$ & & \\
\hline Torsion angles & $\left({ }^{\circ}\right)$ & Torsion angles & $\left({ }^{\circ}\right)$ \\
\hline $\mathrm{N} 1-\mathrm{C} 2-\mathrm{C} 12-\mathrm{C} 11$ & $2.5(4)$ & C3-C2-C12-C13 & $2.8(5)$ \\
\hline C9-N1-C2-C12 & $-177.1(3)$ & $\mathrm{C} 4-\mathrm{C} 3-\mathrm{C} 2-\mathrm{C} 12$ & $176.9(3)$ \\
\hline C7-C8-N21-C22 & $58.1(4)$ & C9-C8-N21-C2C & $70.0(3)$ \\
\hline N21-C8-C7-C6 & $177.8(3)$ & N21-C8-C9-C1C & $-175.9(2)$ \\
\hline
\end{tabular}

C8-N21 bond. The dihedral angles between three planar moieties consisting of two fused-rings are as follows: $\mathrm{AB} / \mathrm{CD}$ 4.2(3) and $\mathrm{AB} / \mathrm{EF} 64.4(3)^{\circ}$. The $\mathrm{AB}$ and $\mathrm{CD}$ moieties make a flat plane with a mean deviation of $0.053(3) \AA$. The O11, O13, $\mathrm{O} 20$ and $\mathrm{O} 22$ atoms lie in the least-square planes calculated from two fused-rings, with mean deviations of 0.011(4), $0.038(5), 0.065(5)$ and $0.064(5) \AA$, respectively. Electron localization was found at the $\mathrm{C} 2-\mathrm{C} 12$ bond with a distance of $1.392(4) \AA$. The planarity of fragment A - D is also stabilized by two asymmetrical intramolecular hydrogen bonds (N1-H…O11 $=2.665(4) \AA$ and $\mathrm{C} 3-\mathrm{H} \cdots \mathrm{O} 13=3.090(4) \AA)$. The fragment $\mathrm{A}-\mathrm{D}$ bonds in-plane at the $\mathrm{C} 2-\mathrm{C} 12$ bond in the direction of the $\mathrm{N} 1$ atom, with the summed bond-angles of N1-C2-C12-C11 and C3-C2-C12-C13 being 240.9(3) and 253.5(4) .

In the crystal structure, there is no significant intermolecular hydrogen bond. The flat A - D fragments are stacked opposed to each other. The solvent molecules insert into the stacked EF fragments perpendicular to the A - D fragments. The solvent molecule sits on a crystallographic center of symmetry, and is disordered in the solvent channels along the $c$-axis.

\section{References}

1. J. V. Malanker, P. Roshan, and G. S. Shankarling, Paintindia, 1996, 46(10), 45.

2. SIR88: M. C. Burla, M. Camalli, G. Cascarano, C. Giacovazzo, G. Polidori, R. Spagna, and D. Viterbo, J. Appl. Cryst., 1989, 22, 389.

3. teXsan, "Single Crystal Structure Analysis Software, Version 1.6", 1993, Molecular Structure Corporation, Texas, USA.

4. ORTEP-3: L. J. Farrugia, J. Appl. Cryst., 1997, 30, 565. 